\title{
A Fuzzy Trust Model Using Multiple Evaluation Criteria
}

\author{
Keon Myung Lee ${ }^{1}$, KyoungSoon Hwang ${ }^{1}$, \\ Jee-Hyong Lee ${ }^{2, \star}$, and Hak-Joon Kim ${ }^{3}$ \\ ${ }^{1}$ School of Electrical and Computer Engineering, \\ Chungbuk National University, Korea \\ ${ }^{2}$ School of Information and Communication Engineering \\ SungKyunKwan University, Korea \\ ${ }^{3}$ Division of Multimedia Information, Howon University, Korea \\ kmlee@cbnu.ac.kr
}

\begin{abstract}
This paper is concerned with a fuzzy trust model which takes into account both evaluations from multiple criteria and the recommendations from others in order to set the trust degrees on entities. In the proposed trust model, the entity's preference degrees on the outcomes of the interactions are expressed in fuzzy sets and the trust degrees are determined by aggregating the satisfaction degrees with respect to evaluation criteria with Sugeno's fuzzy integral. In addition, the reputation information is incorporated into the trust degree determination.
\end{abstract}

\section{Introduction}

Along with the widespread Internet applications such e-commerce, P2P services and so on, the users have no choice but to take some risks in doing transactions with unknown users or systems over the Internet. In everyday life, we estimate the trust degree on the others by considering the past interaction experience with them and sometimes by referring to the reputation, i.e., word of mouth. In the same token, an on-line entity could reduce the risks to run with the help of the trust information for the interacting entities. Even though there have been proposed various trust models[3-10], there are no models yet generally accepted. Some models are qualitative models[3] and others are quantitative models[4,810]. Some models depend only on users' ratings to compute the trust value, and others get the trust values by observing the behaviors of the entity over some period. The trust has been defined in various ways because there are no consensus on what constitutes the trust[3-10]. The following is the Gambetta's[6] which is a well-known definition of trust: Trust (or, symmetrically, distrust) is a particular level of the subjective probability with which an agent will perform a particular action, both before [we] can monitor such action (or independently of his capacity of ever to be able to monitor it) and in a context in which it affects

* Corresponding author. This work was supported by the Regional Research Centers Program of the Ministry of Education \& Human Resources Development in Korea. 
own action. In the trust models, the following three types of trust are usually considered: situational trust, dispositional trust, and general trust. Situational trust (a.k.a., interpersonal trust) is the trust that an entity has for another entity in a specific situation. Dispositional trust (a.k.a, basic trust) is the dispositional tendency of an entity to trust other entities. General trust is the trust of an entity in another entity regardless of situations. On the meanwhile, the reputation is valuable information for estimating the trust of an entity. The trust of an entity can be differently measured according to which aspects we evaluate. Therefore we propose a trust model to consider multiple evaluation criteria and to enable entities to reflect their preference on the outcomes.

The remainder of this paper is organized as follows: Section 2 briefly presents several related works on the trust models. Section 3 briefly explains the $\lambda$-fuzzy measure and the Sugeno's fuzzy integral which are used in the proposed model. Section 4 introduces the proposed fuzzy trust model and Section 5 shows how to apply the model with an example. Finally, Section 6 draws the conclusions.

\section{Related Works}

Trust and reputation have gained great attention in various fields such as economics, distributed artificial intelligence, agent technology, and so on. Various models for trust and reputation have been suggested as a result[3,4,8-10]. Some models just give theoretical guidelines and others provide computational models.

Abul-Rahman et al.[3] proposed a qualitative trust model where trust degrees are expressed in four levels such as very trustworthy, trustworthy, untrustworthy, and very untrustworthy. The model has somewhat ad-hoc nature in defining the trust degrees and the weights. Azzedin et al.[4] proposed a trust model for a peer-to-peer network computing system, which maintains a recommender network that can be used to obtain references about a target domain. The model is specialized for the well-structured network computing system and thus there are some restrictions on applying the model to general cases. Derbas et al.[8] proposed a model named TRUMMAR which is a reputation- based trust model that mobile agent systems can use in order to protect agents from malicious systems. The model pays special attention to use reputation for trust modeling, but does not consider the multiple evaluation criteria. Shi et al.[9] proposed a trust model which uses the statistical information about the possible outcome distribution for actions. In the model, trust is described as an outcome probability distribution instead of a scalar value. When choosing a candidate, it is used to compute the expected utility value for the candidate entities' actions. Wang et al.[10] proposed a trust model based on Bayesian networks for peer-topeer networks. In the model, a Bayesian network is used to represent the trust between an agent and another agent. Such a Bayesian network represents the probabilities to trust an entity in various aspects. The recommendation values from other entities also are incorporated into the model. 


\section{$3 \quad$ Fuzzy Integral for Information Aggregation}

The ability of the fuzzy integral to combine the evaluation results from various perspectives has been shown in several works[1,2]. In order to use fuzzy integral for information aggregation, we should have importance degrees assigned to each powerset element of evaluation criteria. These importance degrees are required to preserve the properties of fuzzy measure[1]. The widely used $\lambda$-fuzzy measure $g_{\lambda}$ satisfies the following property along with the fuzzy measure properties[2]: For a finite set $\left.X=\left\{x_{1}, x_{2}, \ldots, x_{k}\right\}, g_{\lambda}\left(\left\{x_{1}, \ldots, x_{l}\right\}\right)=\frac{1}{\lambda} \prod_{i=1}^{l}\left(1+\lambda g_{i}\right)-1\right]$, where $g_{i}=g_{\lambda}\left(\left\{x_{i}\right\}\right)$.

When all $g_{i}$ s are given, $\lambda$ can be derived from the following equation[1]:

$$
g_{\lambda}(X)=\frac{1}{\lambda}\left[\prod_{i=1}^{t}\left(1+\lambda g_{i}\right)-1\right]=1
$$

Sugeno's fuzzy integral is a Lebesque integral which has the role of aggregating partial evaluations for an entity in consideration of importance degrees of evaluation criteria[1]. Let $X$ be a set of evaluation items and $g(E)$ the importance degree of evaluation criteria set $E \subset X$ with the properties of fuzzy measure. $g(x)$ denotes the evaluation value on the standpoint of evaluation criterion $x$, and $A$ denotes the interest focus of evaluation criteria. The Sugeno's fuzzy integral $\oint_{A} h(x) \circ g(\cdot)$ over the set $A \subset X$ of the function $h$ with respect to a fuzzy measure $g$ is defined as follows:

$$
\begin{gathered}
\oint_{A} h(x) \circ g(\cdot)=\sup _{E \subset X}\left\{\min \left\{\min _{x \in E} h(x), g(A \cap E)\right\}\right\} \\
=\sup _{E \subseteq A}\left\{\min \left\{\min _{x \in E} h(x), g(E)\right\}\right\}
\end{gathered}
$$

Due to the operation $\min _{x \in E} h(x)$, the fuzzy integral has a tendency to produce pessimistic evaluation. Some decision making problem shows that although an item has poor evaluation, the item can be compensated by other good items. Thus to provide the same effect for the fuzzy integral, we can use a compensatory operator $\psi(\{h(x) \mid x \in E\})$ instead of the minimum operator in the operation $\min _{x \in E} h(x)$.

\section{The Proposed Fuzzy Trust Model}

In the literature, there is no consensus on the definition of trust and on what constitutes trust management. In our trust model, however, we take the following definition on the situational trust: Situational trust is the expectation for an entity to provide satisfactory outcomes with respect to the evaluation criteria in a given situation. This section presents how to evaluate the situational trust based on the above definition, how to handle the dispositional trust and the general trust, and how to use recommandation from others and to adjust the recommanders' trust. 


\subsection{Situational Trust}

The situational trust is the trust assigned to an entity for a specific situation. Most existing approaches have interest in how much the considered entity's behaviors are satisfactory [3-10]. It is assumed in their methods to rate the satisfaction degree in a single perspective and it is somewhat ad-hoc on how to rate the satisfaction degrees for an entity to other entities in a specific situation. In the proposed method, the situational trust is estimated as follows: First, an entity $\alpha$ accumulatively constructs the empirical probability distribution of possible outcomes for the interacting entities $\beta$ with respect to each evaluation criterion in the given situation. Each time the entity $\alpha$ needs to measure the trust in another entity $\beta$, she computes the satisfaction degrees with $\beta$ over each evaluation criterion in the situation. After that, the situational trust of $\alpha$ in $\beta$ is determined by aggregating the satisfaction degrees in the perspective of evaluation criteria.

Let $T S_{\alpha}(\beta, \delta ; E C)$ be the situational trust of entity $\alpha$ in entity $\beta$ in the situation $\delta$ with respect to evaluation criteria $E C=\left\{e c_{1}, e c_{2}, \ldots, e c_{n}\right\}$, where $e c_{i}$ is an evaluation criterion. It expresses the degree of expectation for trusted entity $\beta$ to yield satisfactory actions with respect to the evaluation criteria in the given situation. In order to get the situational trust, whenever entity $\alpha$ has an interaction with $\beta, \alpha$ keeps the records about the evaluation outcomes with respect to the evaluation criteria. The evaluation outcomes are given in either continuous values or categorical attributes. In the case of continuous outcomes, the outcome domain is quantized into several prespecified intervals and outcome values are expressed in the corresponding interval labels.

Empirical Outcome Probability Computation. The entity $\alpha$ 's empirical outcome probability for entity $\beta$ to make outcome $o_{i}$ in the situation $\delta$ up to time $t$ with respect to an evaluation criterion $e c_{k}$ is computed as follows:

$$
\begin{gathered}
P^{t}\left(\alpha, \beta, \delta, o_{i} ; e c_{k}\right)=\frac{p^{t}\left(\alpha, \beta, \delta, o_{i} ; e c_{k}\right)}{\sum_{o_{j}} p^{t}\left(\alpha, \beta, \delta, o_{j} ; e c_{k}\right)} \\
p^{t}\left(\alpha, \beta, \delta, o_{i} ; e c_{k}\right)=\rho * \frac{N_{\alpha \beta}^{t}\left(\delta, o_{i} ; e c_{k}\right)}{n_{\alpha \beta}}+(1-\rho) * \frac{N_{\alpha \beta}^{[t-d t, t]}\left(\delta, o_{i} ; e c_{k}\right)}{n_{\alpha \beta}^{[t-d t, t]}}
\end{gathered}
$$

In the above equation, $N_{\alpha \beta}^{t}\left(\delta, o_{i} ; e c_{k}\right)$ indicates the number of outcome $o_{i}$ for $\beta$ to produce with respect to $e c_{k}$ up to time $t, n_{\alpha \beta}$ is the number of total interactions of $\alpha$ with $\beta, N_{\alpha \beta}^{[t-d t, t]}\left(\delta, o_{i} ; e c_{k}\right)$ is the number of outcome $o_{i}$ for $\beta$ to produce with respect to $e c_{k}$ within the recent time window $[t-d t, t], n_{\alpha \beta}$ is the number of outcome $o_{i}$ for $\beta$ to produce with respect to $e c_{k}$ within the window $[t-d t, t]$, and $\rho$ indicates the weighting factor to control the ignorance effect on the past experience.

Satisfaction Degree Computation. The trusting entity $\alpha$ makes her mind on which outcomes are satisfactory for her own preference with respect to each evaluation criterion. For an evaluation criterion $e c_{i}$, suppose that its possible outcome is $P O\left(e c_{i}\right)=\left\{o_{1 i}, o_{2 i}, \ldots, o_{n i}\right\}$. Then, the entity $\alpha$ specifies earlier on her satisfactory outcome set $S O\left(\alpha, e c_{i}\right)$ along with the relative preference for each outcome which is expressed in a fuzzy set as follows: $S O\left(\alpha, e c_{i}\right)=$ 
$\left\{\left(o_{1}, o w_{1}\right), \ldots,\left(o_{j}, o w_{j}\right)\right\}$ where $o_{i} \in P O\left(e c_{i}\right)$ and $w_{k} \in[0,1]$ is the membership degree to indicate the $\alpha$ 's relative preference to the outcome $o_{k}$. The satisfaction degree $S D_{\alpha}\left(\beta, \delta ; e c_{i}\right)$ of $\alpha$ with $\beta$ in the perspective of $e c_{i}$ is determined as follows:

$$
S D_{\alpha}\left(\beta, \delta ; e c_{i}\right)=\sum_{\left(o_{k}, w o_{k}\right) \in S O\left(\alpha, e c_{i}\right)} w o_{k} \cdot P^{t}\left(\alpha, \beta, \delta, o_{k} ; e c_{i}\right)
$$

Situational Trust Computation. In the proposed method, the situational trust is measured by the satisfaction degrees of an entity $\alpha$ with other entity $\beta$ with respect to multiple evaluation criteria $E C$. For example, when a user determines the trust in a restaurant, she considers her satisfaction degrees for it in the point of her own criteria such as taste of food, waiting time to take a table, availability of her favorite food, and so on. The situational trust of $\alpha$ in $\beta$ in situation $\delta$ with respect to evaluation criteria $E C$ is computed as follows: Here, $\psi\left(S D_{\alpha}(\beta, \delta ; A)\right)$ is the value obtained after the application of a compensatory operator to the situational trust values $S D_{\alpha}\left(\beta, \delta ; e c_{i}\right)$ for $e c_{i} \in A$, and $W C(A)$ is the relative importance that $\alpha$ weighs for the evaluation criteria set $A$.

$$
T S_{\alpha}(\beta, \delta ; E C)=\oint_{E C} S D_{\alpha}(\beta, \delta ; \cdot) \circ W C(\cdot)=\sup _{A \subset E C} \min \left\{\psi\left(S D_{\alpha}(\beta, \delta ; A)\right), W C(A)\right\}
$$

\subsection{Dispositional Trust}

The dispositional trust $T D_{\alpha}$ represents the dispositional tendency for a trusting entity $\alpha$ to trust other entities. Each entity is supposed to assign her own dispositional trust value. It could be used as the initial general trust when an entity starts an interaction with a new entity.

\subsection{General Trust}

The general trust $T G_{\alpha}(\beta)$ of entity $\alpha$ in entity $\beta$ is the trust that $\alpha$ has on $\beta$ regardless of situations. It plays the role of the initial situational trust for $\beta$ in a new situation. It can be used as the reputation weight for $\beta$ at the beginning. It can be also used as the situational trust value while enough interactions have not yet made. The general trust of $\alpha$ in $\beta$ is obtained by averaging the situational trusts for the experienced situations $\Phi$ as follows:

$$
T G_{\alpha}(\beta)=\frac{\sum_{\delta \in \Phi} T S_{\alpha}(\beta, \delta ; E C)}{|\Phi|}
$$

\subsection{Reputation}

When an entity decides whether it starts an interaction with another entity, it is valuable to refer to available reputation information about the entity. A reputation is an expectation about an entity's behavior which is formed by the community having interacted with the entity based on the information about or the observations of its past behaviors. 
When the recommenders $\gamma_{j}$ are available for an entity $\beta$ in a situation $\delta$, the entity $\alpha$ might take into account the recommendations for $\beta$. Each entity could have different preference on the outcomes with respect to the evaluation criteria and thus it is not so meaningful to directly use the trust values for $\beta$ from the recommenders $\gamma_{j}$. Therefore we take the approach to take the recommender $\gamma_{j}$ 's empirical outcome probabilities $P^{t}\left(\gamma_{j}, \beta, \delta, o_{k} ; e c_{i}\right)$ for $\beta$ instead of $\gamma_{j}$ 's trust value $T S_{\gamma_{j}}(\beta, \delta ; E C)$ on $\beta$. With the received outcome probabilities, the satisfaction degrees $S D_{\gamma_{j}}^{r}\left(\beta, \delta ; e c_{i}\right)$ from $\gamma_{j}$ are computed as follows: Here $w o_{k}^{\alpha}$ is the preference degree of the entity $\alpha$ for the outcome $o_{k}$.

$$
S D_{\gamma_{j}}^{r}\left(\beta, \delta ; e c_{i}\right)=\sum_{o_{k} \in S O\left(\alpha, e c_{i}\right)} w o_{k}^{\alpha} \cdot P^{t}\left(\gamma_{j}, \beta, \delta, o_{k} ; e c_{i}\right)
$$

Then the situational trust value $T S_{\gamma_{j}}^{r}(\beta, \delta ; E C)$ from the recommender $\gamma_{j}$ is computed as follows: Here $\psi\left(S D_{\gamma_{j}}^{r}(\beta, \delta ; A)\right)$ is the value obtained by the application of a compensatory operator to the satisfaction degrees $S D_{\gamma_{j}}^{r}\left(\beta, \delta, e c_{k}\right)$ for $e c_{k} \in A$ from $\gamma_{j}$, and $W C(A)$ is the relative importance that $\alpha$ weighs for the evaluation criteria set $A$.

$$
T S_{\gamma_{j}}^{r}(\beta, \delta ; E C)=\oint_{E C} S D_{\gamma_{j}}^{r}(\beta, \delta ; \cdot) \circ W C(\cdot)=\sup _{A \subset E C} \min \left\{\psi\left(S D_{\gamma_{j}}^{r}(\beta, \delta ; A)\right), W C(A)\right\}
$$

The reputation value $T R_{\alpha}(\beta, \delta ; E C)$ of $\beta$ for $\alpha$ is computed by taking the weighted sum of the situational trust $T S_{\gamma_{j}}^{r}(\beta, \delta ; E C)$ from recommenders $\gamma_{j}$ as follows: Here the weighting factor $w r_{j}$ is the recommendation trust value for the recommender $\gamma_{j}$. That is, $w r_{j}$ is the degree to which $\alpha$ believes the recommendation from $\gamma_{j}$. These weights are updated through the interaction with the entities.

$$
T R_{\alpha}(\beta, \delta ; E C)=\frac{\sum_{j} w r_{j} \cdot T S_{\gamma_{j}}^{r}(\beta, \delta ; E C)}{\sum_{j} w r_{j}}
$$

\subsection{Combination of Situational Trust and Reputation}

When an entity starts to work in a community, she assigns her own dispositional trust value. The dispositional trust is used as the initial general trust when she interacts with an entity for the first time. Until sufficient number of interactions has made for a given situation, the general trust is used as the situational trust. Once the situational trust $T S_{\alpha}(\beta, \delta ; E C)$ and the reputation $T R_{\alpha}(\beta, \delta ; E C)$ are obtained, the final trust value $T S_{\alpha}(\beta, \delta ; E C)$ is computed by their weighted aggregation as follow: Here, $w$ is the relative weighting factor for the situational trust, $w \in[0,1]$.

$$
T S_{\alpha}(\beta, \delta ; E C)=w \cdot T S_{\alpha}(\beta, \delta ; E C)+(1-w) \cdot T R_{\alpha}(\beta, \delta ; E C)
$$

\subsection{Update of the Recommender Trust}

The recommender's trust $w r_{i}$ is updated according to how much their recommendation score is close to the final computed trust value $T S_{\alpha}(\beta, \delta ; E C)$. If 
the recommendation score is similar to the final trust value, the recommender's recommendation trust is increased by a small amount. Otherwise, the recommender's recommendation trust is decreased by an exponential factor term. The following shows the update rule for the recommender trust $w r_{i}$.

Let $\Delta=\left|T S_{\alpha}(\beta, \delta ; E C)-T S_{\gamma_{i}}^{r}(\beta, \delta ; E C)\right|$. If $\Delta<\epsilon, w r_{i}(t+1)=\min \left\{w r_{i}(t)\right.$. $(1+\eta), 1\}$ where $\epsilon$ and $\eta$ are small values such that $0 \leq \epsilon, \eta \leq 1$. Otherwise, $w r_{i}(t+1)=w r_{i}(t)\left(1-e^{-\lambda \Delta}\right)$ where $\lambda$ is a small value such that $0 \leq \lambda \leq 1$.

\section{An Application Example}

In order to show how the proposed model works, the section gives an example to apply the model. Suppose that an entity $P_{1}$ has interest in the trust of a restaurant $R_{1}$ in terms of $E C=\{$ taste $t s$, waiting time $w t$, favorite food availability $f a\}$ with the help of the recommenders $P_{2}$ and $P_{3}$. Suppose that the satisfying outcome sets of $P_{1}$ for $E C$ are given in the following fuzzy sets:

$$
\begin{aligned}
& S O(t s)=\{(\text { bad } b d, 0),(\text { moderate } m d, 0.5),(\text { good } g d, 0.7),(\text { excellent } e x, 1)\} \\
& S O(w t)=\left\{\left([0,15] t_{1}, 1\right),\left((15,30] t_{2}, 0.7\right),\left((30,50] t_{3}, 0.5\right),\left((50, \infty) t_{4}, 0\right)\right\} \\
& S O(f a)=\{(\text { available } a v, 1),(\text { not available } n a, 0)\}
\end{aligned}
$$

Let the situation $\delta$ be going out to the restaurant $R_{1}$ on the weekends. Suppose that the empirical outcome probability distributions $P^{t}\left(P_{i}, R_{1}, \delta, o_{i} ; e c_{k}\right)$

\begin{tabular}{|c|c|c|c|c|c|c|c|c|c|c|}
\hline & \multicolumn{10}{|c|}{$o_{i}$} \\
\hline & \multicolumn{4}{|c|}{ taste $t s$} & \multicolumn{4}{|c|}{ waiting time $w t$} & \multicolumn{2}{|c|}{ availability $f a$} \\
\hline & $b d$ & $\overline{m d}$ & $g d$ & $e x$ & $t_{1}$ & $t_{2}$ & $t_{3}$ & $t_{4}$ & $a v$ & $n a$ \\
\hline$P^{t}\left(P_{1}, R_{1}, \delta, o_{i} ; E C\right)$ & 0 & 0.2 & 0.3 & 0.5 & 0.7 & 0.1 & 0.2 & 0 & 0.6 & 0.4 \\
\hline$P^{t}\left(P_{2}, R_{1}, \delta, o_{i} ; E C\right)$ & 0.2 & 0.2 & 0.5 & 0.1 & 0.4 & 0.5 & 0.1 & 0 & 0.5 & 0.5 \\
\hline$P^{t}\left(P_{3}, R_{1}, \delta, o_{i} ; E C\right)$ & 0 & 0.1 & 0.2 & 0.7 & 0.3 & 0.4 & 0.2 & 0.1 & 0.7 & 0.3 \\
\hline
\end{tabular}
are given as in Table 1:

Table 1. The empirical outcome probability distributions

Computation of the Trust. Then the satisfaction degree of $P_{1}$ and the satisfaction degrees from $P_{2}$ and $P_{3}$ in perspective of $P_{1}$ is obtained using Eq.(6) and Eq.(9) respectively, as in Table 2.

Table 2. The satisfaction degrees

\begin{tabular}{c|c|c|c}
\hline & \multicolumn{3}{|c}{$e c_{i}$} \\
\cline { 2 - 4 } & taste $t s$ & waiting time $w t$ & availability $f a$ \\
\hline$S D_{P_{1}}\left(R_{1}, \delta ; e c_{i}\right)$ & 0.84 & 0.87 & 0.6 \\
$S D_{P_{2}}^{r}\left(R_{1}, \delta ; e c_{i}\right)$ & 0.6 & 0.8 & 0.5 \\
$S D_{P_{3}}^{r}\left(R_{1}, \delta ; e c_{i}\right)$ & 0.91 & 0.68 & 0.7 \\
\hline
\end{tabular}


Suppose that the relative importance degrees for the evaluation criteria are given as $0.5,0.3$, and 0.2 to $t s, w t$, and $f a$, respectively, and the importance degrees satisfy the properties of the $\lambda$-fuzzy measure. From Eq.(9), we can get a parameter $\lambda$ of the $\lambda$-fuzzy measure satisfying $0.06 \lambda^{2}+0.47 \lambda+0.2=0$. The unique root greater than -1 for this equation is $\lambda=-0.45$ which produces the following fuzzy measure on the power set of $E C$.

\begin{tabular}{c|c}
\hline subset $A$ of $E C$ & $g_{-0.45}(A)$ \\
\hline$\phi$ & 0 \\
$\{t s\}$ & 0.5 \\
$\{w t\}$ & 0.3 \\
$\{f a\}$ & 0.4 \\
$\{t s, w t\}$ & 0.87 \\
$\{w t, f a\}$ & 0.75 \\
$\{f a, t s\}$ & 0.99 \\
$\{t s, w t, f a\}$ & 1 \\
\hline
\end{tabular}

For Eq.(7), let us use the compensatory operator $\psi(A)=\tau \cdot \min _{x_{i} \in A}\left\{x_{i}\right\}+$ $(1-\tau) \cdot \max _{x_{i} \in A}\left\{x_{i}\right\}$ where $\tau=0.4$. Then the situational trust $T S_{P_{1}}\left(R_{1}, \delta ; E C\right)$ of $P_{1}$ in $R_{1}$ is computed by Eq.(7) as follows:

$$
\begin{aligned}
& T S_{P_{1}}\left(R_{1}, \delta ; E C\right)=\sup _{A \subset E C} \min \left\{\psi\left(S D_{P_{1}}\left(R_{1}, \delta ; A\right)\right), W C(A)\right\} \\
& =\sup \{\min \{0.84,0.5\}, \min \{0.6,0.3\}, \min \{0.91,0.4\}, \min \{0.744,0.87\}, \\
& \quad \min \{0.786,0.75\}, \min \{0.882,0.99\}, \min \{0.84,1\}\}=0.882
\end{aligned}
$$

The situational trusts $T S_{P_{i}}^{r}\left(R_{1}, \delta ; E C\right)$ from the recommenders $P_{2}$ and $P_{3}$ are computed by Eq.(10) as follows:

$$
T S_{P_{2}}^{r}\left(R_{1}, \delta ; E C\right)=0.89 \quad T S_{P_{3}}^{r}\left(R_{1}, \delta ; E C\right)=0.80
$$

If the recommender trust values $w r_{P_{i}}$ for $P_{2}$ and $P_{3}$ are 0.8 and 0.7 , respectively, then the reputation of $R_{1}$ for $P_{1}$ is computed as follows:

$$
T R_{P_{1}}\left(R_{1}, \delta ; E C\right)=(0.8 * 0.89+0.7 * 0.80) /(0.8+0.7)=0.837
$$

If the weighting factor $w$ for the situation trust is 0.7 , then the final trust value $T S_{P_{1}}\left(R_{1}, \delta ; E C\right)$ is computed by Eq.(12) as follows:

$$
T S_{P_{1}}\left(R_{1}, \delta ; E C\right)=0.7 * 0.882+0.3 * 0.837=0.867
$$

Based on this trust value, the entity $P_{1}$ would decide whether to do business with the entity $R_{1}$.

\section{Conclusions}

The trust information for the online entities are valuable in reducing the risks to take on doing some transactions. We proposed a fuzzy trust model which has the following characteristics: The model allows to look at entity's trust in the point of multiple evaluation criteria. It maintains the empirical outcome distributions for evaluation criteria and enables the trusting entities to express 
their fuzzy preference on the outcomes when estimating trust in other entities. In addition, the model makes it possible for the entities to put different weights on the evaluation criteria, which are aggregated by using Sugeno's fuzzy integral. When it makes use of the recommendations from others, it takes the outcome distributions instead of their recommending trust values. Thereby, it allows to reflect the trusting entity's preference and her own weighting on the evaluation criteria in the evaluation of the recommendation.

\section{References}

[1] H.-J. Zimmermann. Fuzzy Set Theory and its Applications. Kluwer-Nijhoff Publishing. 364p. (1985).

[2] K. M. Lee, H. Lee-Kwang. Information Aggregating Networks based on Extended Sugeno's Fuzzy Integral. LNCS 1011. 56-66. (1995).

[3] A. Abdul-Rahman, S. Hailes. Supporting Trust in Virtual Communities. Proc. of the Hawaii Int. Conf. on System Sciences. (Jan.4-7, 2000, Maui Hawaii).

[4] F. Azzedin, M. Maheswaran. Trust Modeling for Peer-to-Peer based Computing Systems. Proc. of the Int. Parallel and Distributed Processing Symposium. (2003).

[5] U. Hengartner, P. Steenkiste. Implementing Access Control to People Location Information. Proc. of SACMAT'04. (Jun.2-4, 2004. New York).

[6] D. Gambetta. Can We Trust Trust?. In Trust: Making and Breaking Cooperative Relations.(Gambetta. D (ed.)). Basil Blackwell. Oxford. (1990).

[7] D. H. McKnight, N.L. Chervany. The Meanings of Trust. Technical Report 94-04. Carlson School of Manangement, University of Minnesota. (1996).

[8] G. Derbas, A. Kayssi, H. artial, A. Cherhab. TRUMMAR - A Trust Model for Mobile Agent Systems Based on Reputation. In Proc. of ICPS2004. IEEE. (2004).

[9] J. Shi, G. v. Bochmann, C. Adams. A Trust Model with Statistical Foundation. In FAST'04. Academic Press. (2004).

[10] Y. Wang, J. Vassileva. Bayesian Network Trust Model in Peer-to-Peer Network. In Proc. of WI'03. IEEE. (2003). 